\title{
Aerothermal Analysis of a Turbine Casing Impingement Cooling System
}

\author{
Riccardo Da Soghe, Bruno Facchini, Mirko Micio, and Antonio Andreini \\ Energy Engineering Department “S. Stecco”, University of Florence, Street S. Marta 3, 50139 Florence, Italy \\ Correspondence should be addressed to Riccardo Da Soghe, dasoghe@brun.de.unifi.it \\ Received 19 July 2012; Revised 28 September 2012; Accepted 30 September 2012 \\ Academic Editor: N. Sitaram
}

Copyright ( $) 2012$ Riccardo Da Soghe et al. This is an open access article distributed under the Creative Commons Attribution License, which permits unrestricted use, distribution, and reproduction in any medium, provided the original work is properly cited.

\begin{abstract}
Heat transfer and pressure drop for a representative part of a turbine active cooling system were numerically investigated by means of an in-house code. This code has been developed in the framework of an internal research program and has been validated by experiments and CFD. The analysed system represents the classical open bird cage arrangement that consists of an air supply pipe with a control valve and the present system with a collector box and pipes, which distribute cooling air in circumferential direction of the casing. The cooling air leaves the ACC system through small holes at the bottom of the tubes. These tubes extend at about $180^{\circ}$ around the casing and may involve a huge number of impinging holes; as a consequence, the impinging jets mass flow rate may vary considerably along the feeding manifold with a direct impact on the achievable heat transfer levels. This study focuses on the performance, in terms of heat transfer coefficient and pressure drop, of several impinging tube geometries. As a result of this analysis, several design solutions have been compared and discussed.
\end{abstract}

\section{Introduction}

Impingement with high velocity jets has become an established method for surface cooling or heating in a wide variety of processes and thermal control applications. The use of impingement jets for the cooling of modern aeroengine components is widespread, especially within the hot stationary parts. Since the cooling performance of impinging jets is very high, this method provides an efficient way to manage a component heat load when a sufficient pressure head and geometrical characteristics are available for its implementation. The cooling jets are usually arranged as arrays [1].

Aero-engine casing temperature control is a very effective way to reduce aerodynamic losses and specific fuel consumption due to blade tip clearance. Because of the significant variations of centrifugal and thermal loads which occur at different engine operating conditions, the tip clearance can be extremely variable; such dimensional variation may worsen engine performance and reduce the components' life span [2]. To overcome these issues, the Active thermal Clearance Control (ACC), generally based on impingement cooling, has been successfully introduced in several applications as described in Halila et al. [3], Beck and Fasching [4], and more recently by Justak and Doux [2]. In such systems, impinging jets are directed towards the external turbine casing by means of a series of circumferential feeding pipes with the final aim of keeping the clearance between blade tip and casing as constant as possible under different engine operating conditions (Figures 1 and 2). Depending on the engine architecture, the cooling air could be bled from the compressor or extracted from the fan flow. The impinging system pressure ratio is then a consequence of the ACC configuration: lower than 1.1 in case of cooling air extracted from the fan flow, while higher $\beta$ when the coolant comes from the compressor.

The correct evaluation of both the impinging jet mass flow rate (i.e., the characterization of the holes discharge coefficient) and the related heat transfer coefficient is a fundamental activity.

The discharge coefficient $\left(C_{d}\right)$ is defined as the ratio of the actual mass flow rate through a hole and the isentropic flow rate. It summarizes all the losses that limit the actual 


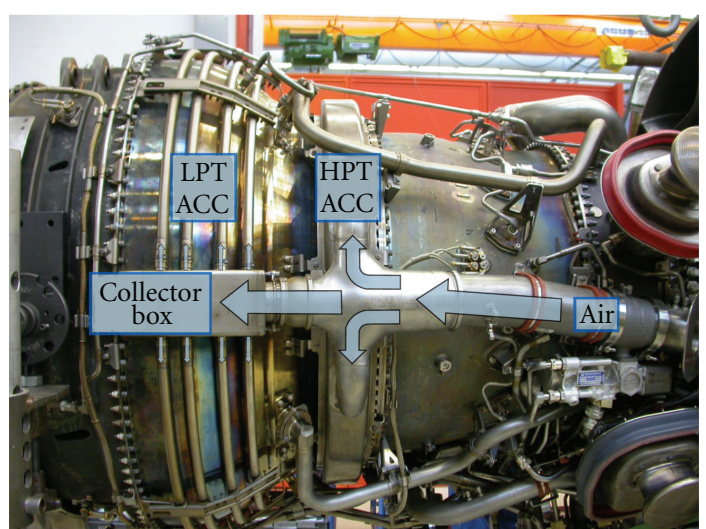

Figure 1: ACC system, Ahmed et al. [5].

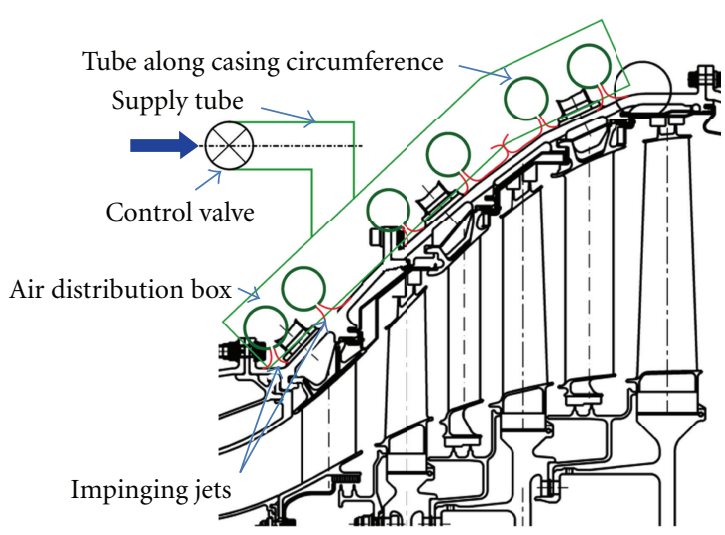

Figure 2: Scheme of a LPT ACC system, Ahmed et al. [6].

mass flow rate through a hole: entry pressure losses, internal losses due to friction, and exit losses.

Many parameters may influence the discharge coefficient [7]: geometrical, such as hole shape, hole angle, space between holes, and length to diameter ratio, and fluiddynamical, such as pressure ratio across the hole and Reynolds and Mach number of the two cross-flows and inside the hole. For this reason, several studies have been carried out on different geometries of holes subjected to a wide range of fluid-dynamics conditions; extensive reviews can be found in Hay and Lampard [8] and in Andreini and DaSoghe [9]. Gritsch et al. [10] have investigated the behaviour of a single hole of large diameter $(10 \mathrm{~mm})$; they proposed a method for correlating the discharge coefficients, assuming that pressure losses inside the hole and those related to the hole entry and exit are independent. Internal losses are found to be dependent on the pressure ratio across the hole, while the entry and exit losses depend on the jet to cross-flow momentum ratio. With these hypotheses in mind, Gritsch et al. [11] have studied the influence of the internal cross-flow on shaped holes, while Rowbury et al. [12] proposed a method to quantify the influence of the external cross-flow on the $C_{d}$. More recently, Schulz et al. [13] have performed several experiments, analysing the behaviour of the $C_{d}$ under varying internal and external cross-flow conditions and geometrical angles and using the jet-to-cross-flow momentum ratio to reduce data.

Referring to the heat transfer, measurements of multiple jet impingement arrays can be found in a relevant number of existing publications. Comprehensive reviews on this field have been provided by Martin [15] and Han et al. [16]. Some recent and very interesting contributions have been made by Ahmed and coworkers [5, 6] who have performed some numerical simulations of the flow in a short tube section of an ACC system for a low pressure turbine. The length-to-diameter ratio of the sharp-edged cylindrical nozzles, ranging from 0.25 to 2, was also accounted for. The authors reveal that increasing the Mach number by simultaneously reducing the orifice diameters led to slightly decreasing Nusselt numbers, with average deviations of the order of $14 \%$. The predicted discharge coefficients increased significantly by augmenting the Mach number. The main limiting aspect of these two interesting contributions consists in the fact that the authors do not evaluate the effects of the undercowl flow on the impingement jets.

As mentioned above, many published works deal with heat transfer coefficient due to impinging jets; most of them analyse, by means of experiments or numerical simulations, the behaviour of several hole geometries and arrangements, but it also appears a complete deficiency of studies conduced on a real-engine ACC geometry. Similar conclusions could be pointed out referring to impinging holes discharge coefficient prediction. Indeed, there seems to be a lack of general studies, and therefore of useful design correlations concerning the effects on the discharge coefficient due to the interaction among adjacent holes drilled in cylindrical feeding pipes [9].

Hence, the University of Florence has promoted an internal research project aimed at the characterization of the holes discharge coefficient and heat transfer phenomena of a real-engine ACC geometry. The main goals of this project are to provide a general understanding of the fluid flow and heat transfer characteristics, to develop useful correlations for both the $C_{d}$ and the HTC coefficient and, finally, to validate CFD with high quality experimental data. In the framework of the project two test rigs have been developed. The first one, aims to evaluate the discharge coefficient of several jet holes drilled into a circular duct. The tested geometries replicate the final part of a feeding manifold impingement tube used in active clearance control systems. By using the provided experimental data (see [17]) and validated CFD calculations, Andreini and DaSoghe [9] have developed an empirical correlation for the prediction of the impinging holes discharge coefficient: it expresses the $C_{d}$ of each hole as a function of the ratio between the hole and the manifold mass velocity and the local value of the pressure ratio. Recently, the authors have revised this correlation to make it sensible to the nozzle length-todiameter ratio [18]. The second test rig, aims to evaluate the heat transfer coefficient and the adiabatic effectiveness of a multijet impingement array (for which the undercowl flow is considered) which reproduces an active clearance control system of a commercial aircraft. The test rig has 


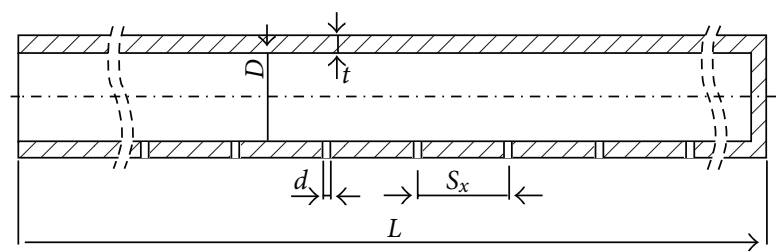

FIGURE 3: Multiple jet section geometry.

provided detailed experimental data that are, nowadays, partially available in the open literature (see [14]).

This paper deals with a simplified 1D code, developed at the University of Florence, aimed at the design of the multiple jet section of an ACC cooling tube. The main purpose of this work is to validate the in-house design tool and, by using of its predictions, to point out design practices for such kind of cooling systems.

\section{Description of the Code}

As stressed above, the simplified code deals with the multiple jet section of an ACC cooling tube. Such kind of geometries are depicted in Figure 3.

The aim of the $1 \mathrm{D}$ program is to evaluate the mass flow rate through each nozzle of the array and then to calculate the related heat transfer coefficient HTC achieved on the target surface. To do that the code, that is based on a correlative approach, implements two correlations: the first one for the nozzle $C_{d}$ and the other one for the HTC evaluation. The implemented correlations are presented in the next section. Within the manifold, the total pressure losses due to friction are neglected. Then, the static pressure profile is determined by the mass flow rate bleeding operated by the holes.

\section{Discharge Coefficient Correlation}

The correlation for the $C_{d}$ parameter considered in this study has been recently developed by DaSoghe and Andreini $[9,18]$. The expression is the result of an extensive CFD analysis: it expresses the $C_{d}$ of each impingement hole as a function of the ratio between the hole and the manifold mass velocity (MVR), the nozzle length-to-diameter ratio $(t / d)$ and the local value of the $\beta_{L}$ ratio. The discharge coefficient is evaluated using the following expression:

$$
C_{d}=C_{1} \cdot \beta_{L}^{\alpha(t / d)} \cdot g\left(\frac{t}{d}\right) \cdot\left(1+C_{2} \cdot C_{3}^{C_{4} \cdot \mathrm{MVR}^{C_{5}}}\right)
$$

where $\alpha(t / d)$ and $g(t / d)$ are second-order polynomial functions of the length-to-diameter ratio and the other coefficients are suitable constants.

Even developed referring to circular cross-section manifold geometries, DaSoghe and Andreini [18] proved the correlation reliability also in case of squared manifold geometries.

The ranges of applicability of the correlation are the followsing $\beta=1.0$ to $1.65, \operatorname{Re}_{\text {channel }}=0$ to $6 \cdot 10^{4}$, and $t / d=0.25$ to 3 . With respect to the CFD data, the correlation
TABLE 1: Geometric parameter.

\begin{tabular}{lc}
\hline Manifold diameter "D" & $\mathrm{m}$ \\
Nozzle diameter " $d$ " & $\mathrm{m}$ \\
Manifold thickness " $t$ " & $\mathrm{m}$ \\
Holes number " $n$ " & - \\
Holes spacing "Sx" & $\mathrm{m}$ \\
Nozzle to wall distance " $h$ " & $\mathrm{m}$ \\
\hline
\end{tabular}

TABLE 2: Thermodynamic parameter.

Cooling temperature $\mathrm{K}$

Wall temperature $\quad \mathrm{K}$

Overall pressure ratio

Discharge pressure $\quad \mathrm{Pa}$

shows a mean relative error of $1.6 \%$ and a maximum error of $4.5 \%$, with a standard deviation of $1.2 \%$ (i.e., $95 \%$ of the $C_{d}$ predicted when using the correlation leads to an error lower than $3 \%$ with respect to the CFD data set).

Finally, the correlation has been validated against the experimental data provided by Schulz and co-workers [13].

For further details about the presented correlation and the related validation study, refer to Andreini and DaSoghe papers $[9,18]$.

\section{Heat Transfer Coefficient Correlation}

The method used to calculate the HTC consists in a procedure that considers two correlations: one to evaluate the HTC peak value on the jet stagnation point, and the other to calculate the HTC value far away from the stagnation region.

The HTC peak value is evaluated using of the following expression:

$$
\mathrm{Nu} 0=C_{6} \operatorname{Re}_{d}^{C_{7}} \operatorname{Pr}^{C_{8}}\left(\frac{S_{x}}{d}\right)^{C_{9}}\left(\frac{h}{d}\right)^{C_{10}}
$$

in which the $\mathrm{Nu} 0$ represents the Nusselt number peak value (i.e., the $\mathrm{Nu}$ at the jet stagnation point). This expression has been derived from Florschuetz and co-workers [19]. The ranges of applicability of the correlation are the following: $\operatorname{Re}_{d}=2500$ to $80000, S_{x} / d=5.9$ to 31.4 , and $h / d=2.9$ to 17.8. Far away from the stagnation region, the local Nusselt number is evaluated as follow:

$$
\mathrm{Nu} l=\mathrm{Nu} 0\left[1-C_{11}\left(\frac{x}{d}\right)^{C_{12}}\right] \exp \left[C_{13}\left(\frac{x}{d}\right)^{C_{14}}\right],
$$

where $\mathrm{Nu} 0$ is the Nusselt number evaluated by (2) and $x$ represents the distance between the considered point and the stagnation point.

\section{5. $1 D$ Procedure}

The in-house code is composed of two different modules. The first one consists in a performance code: for a given ACC tube geometry and operating condition (see Tables 1 and 2), 
TABle 3: Design code: Geometric parameter.

\begin{tabular}{lc}
\hline Manifold diameter " $D$ " & $\mathrm{m}$ \\
Manifold thickness " $t$ " & $\mathrm{m}$ \\
Holes number " $n$ " & - \\
Holes spacing "Sx" & $\mathrm{m}$ \\
Nozzle to wall distance " $h$ " & $\mathrm{m}$ \\
\hline
\end{tabular}

TABLE 4: Validation of the 1D code: Analized geometries.

\begin{tabular}{ccccccc}
\hline & $D$ & $d$ & $n$ & $t / d$ & $\mathrm{Sx} / d$ & $h / d$ \\
\hline Geom 1 & $12(\mathrm{~mm})$ & $1(\mathrm{~mm})$ & 17 & 2 & 12 & 7 \\
Geom 2 & $12(\mathrm{~mm})$ & $1(\mathrm{~mm})$ & 133 & 2 & 1.5 & 7 \\
\hline
\end{tabular}

the code evaluates the system performance in terms of heat transfer coefficient distribution and HTC mean value on the target surface.

By means of an iterative procedure, the code first evaluates the isentropic mass flow rate through the generic impingement hole, using the St. Venant equation:

$$
\dot{m}_{\text {ideal }}=\frac{P \cdot A}{\sqrt{R \cdot T}} \cdot \sqrt{\frac{2 \gamma}{\gamma-1}\left[\left(\frac{1}{\beta_{L}}\right)^{1 / \gamma}-\left(\frac{1}{\beta_{L}}\right)^{\gamma+1 / \gamma}\right]} .
$$

The actual mass flow rate through a generic hole of the array is then calculated using the following expression:

$$
\dot{m}_{\text {actual }}=\dot{m}_{\text {ideal }} \cdot C_{d} \text {, }
$$

where the $C_{d}$ is given in (1). Once the $\dot{m}_{\text {actual }}$ is evaluated, the code calculates the jet heat transfer coefficient distribution.

The second code's module, consists in a design procedure: starting from some geometrical data and operating conditions (i.e., overall pressure ratio), the code determines the nozzles' diameter in order to obtain a desired level of heat transfer coefficient. In this case the requested geometrical parameters are reported in Table 3.

The procedure starts from the last hole of the array (the hole closest to the manifold endcap) for which the MVR is $\infty$. In that case the hole $C_{d}$ is function of both the pressure ratio and the $t / d$ ratio thus it can be easily estimated. Once the $C_{d}$ and the isentropic mass flow rate are calculated, the code estimates the hole impinging mass flow rate and then the related mean HTC. If the calculated HTC does not match the imposed one, the code assumes a new hole diameter and then recalculates the $C_{d}$, the isentropic mass flow rate and the jet mean HTC. These steps are repeated until the calculated mean HTC matches the desired value. Once the procedure is converged for the last hole of the array, the code starts to analyse the second last hole of the array and so on.

\section{Validation of the Code}

In order to evaluate the accuracy and reliability of the code, the predictions of the 1-D procedure are compared with the experimental and numerical data provided by Facchini et al. [14] and Andreini and DaSoghe [9]. Two geometries have been considered (Table 4).

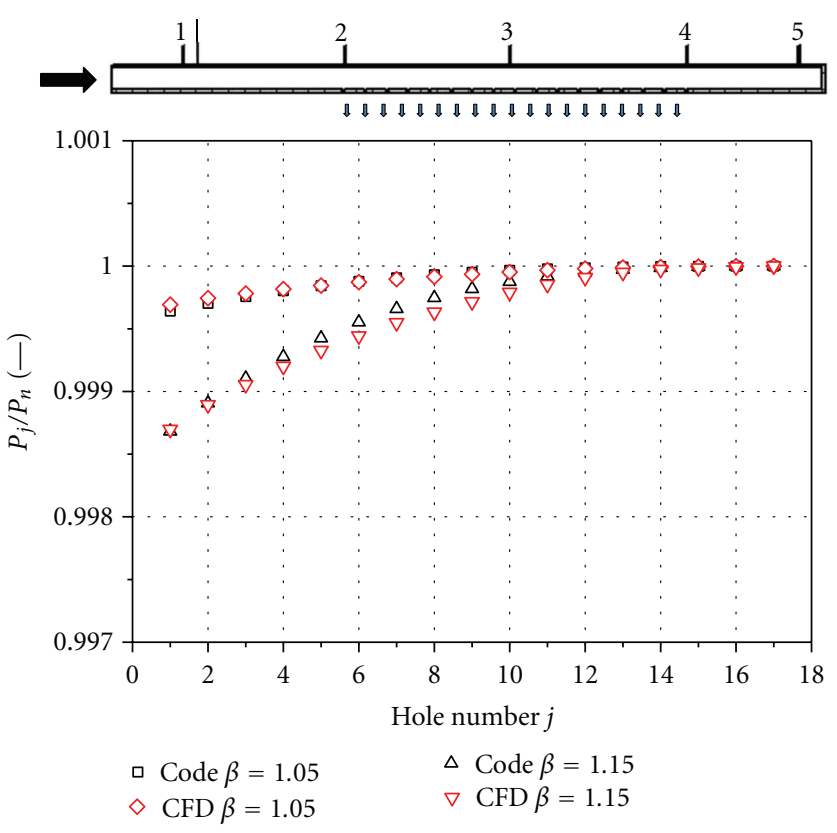

Figure 4: Pressure distribution for Geom 1.

The comparisons made for two operating conditions ( $\beta=1.05$ and $\beta=1.15)$, are provided in terms of pressure distribution (expressed as the ratio of the the value in correspondence of the generic hole and value at the end of the feeding pipe $\left.P_{j} / P_{n}\right)$, mass flow rate split along the manifold (expressed as the ratio of the generic hole's mass flow rate and the mass flow rate at the inlet of the feeding pipe $\left.m_{j} / m_{\text {tot }}\right)$ and HTC profiles.

Figures 4 and 5 show the pressure distribution and the mass flow rate split across the manifold for the geometry labelled "Geom 1".

The code predictions are compared with the CFD data provided by Andreini and DaSoghe [9]. As shown by the figures, the 1-D code well agrees with the CFD for all the tested operating conditions meaning that a reliable pressure and hole mass flow rates evaluation is provided.

The HTC profiles calculated by the in-house program for the central nozzle of the array are compared with the related experimental data of Facchini et al. [14]. The mentioned test rig consists in a real engine geometry that counts several ACC manifolds. The presence of the undercowl flow is also accounted for during the experiments. The results compared here consist in the spanwise HTC profiles (Figure 6). In the experiments, heat transfer coefficients were determined by means of a steady state technique, measuring wall temperatures on an heated surface, using TLC (thermo liquid crystal) paint. Maximum relative error referred to the heat transfer measurement is under $\pm 12 \%$. For further details about the experimental procedure and results, please refer to Facchini et al. [14].

In Figure 7, the HTC profile predicted by the simplified procedure in case of Geometry 1 is compared with Facchini et al.s [14] experimental data. 


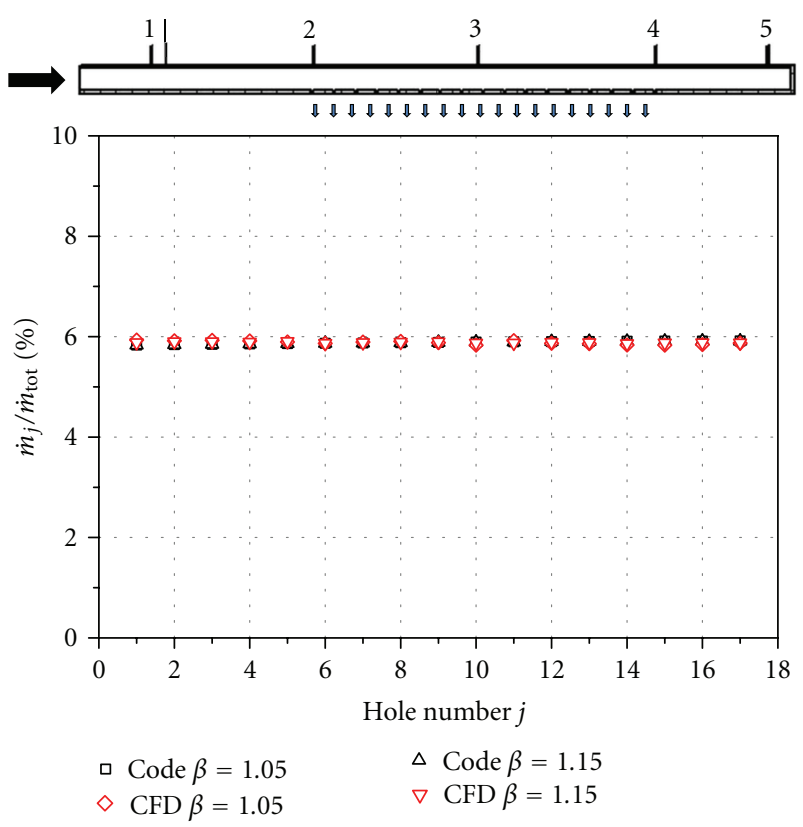

Figure 5: Mass flow split distribution for Geom 1 (percent).

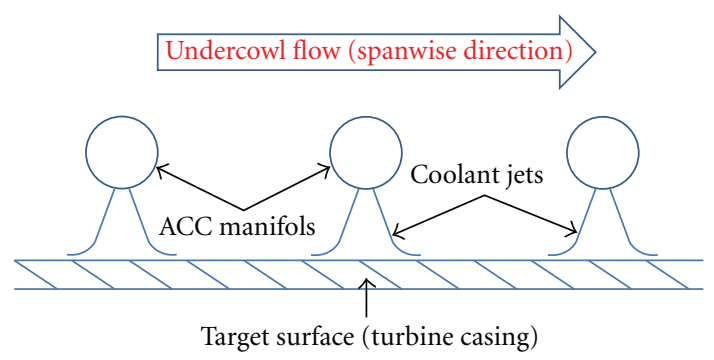

Figure 6: Spanwise direction.

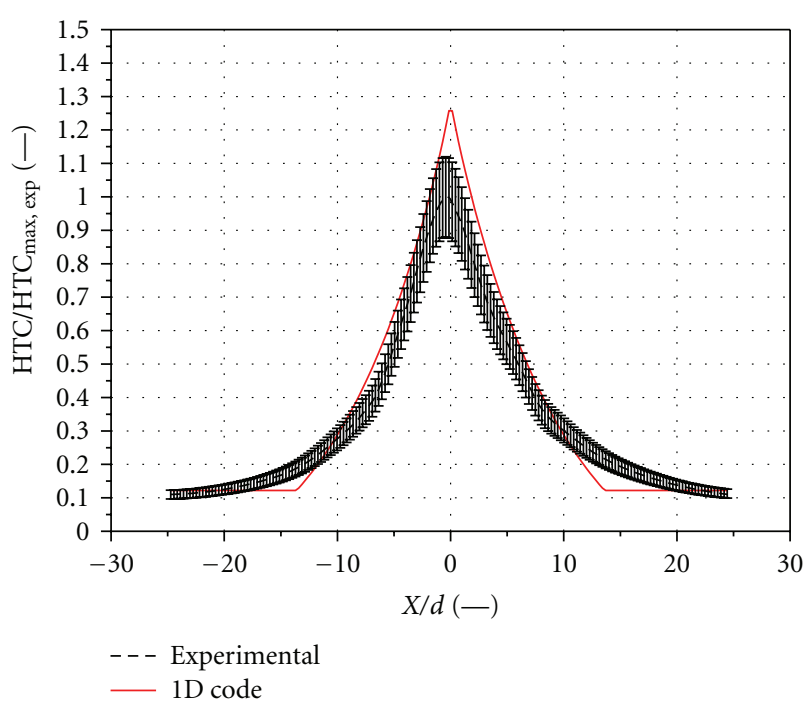

Figure 7: Heat transfer coefficient distribution, central hole of the array, Geom $1 \beta=1.15$ (experimental data from Facchini et al. [14]).

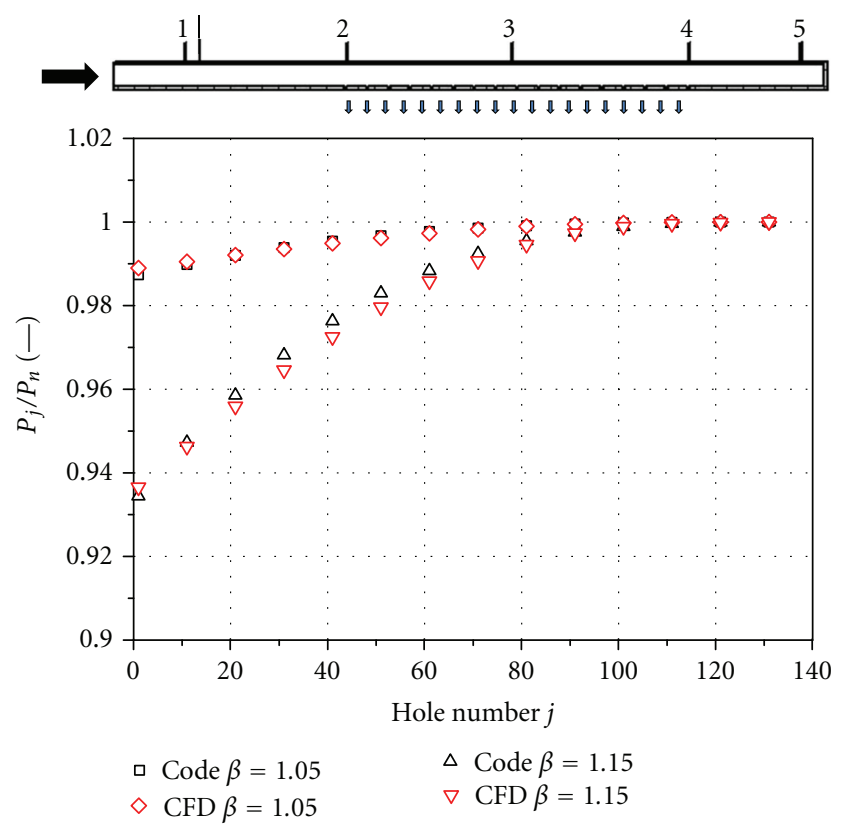

Figure 8: Pressure distribution for Geom 2.

It emerges from the figures that the code is in fairly good agreement with the experiments in term of HTC profiles. The agreement becomes better when considering HTC mean values (that are the key parameter we are looking at). Similar conclusions can be drawn also for the geometry labelled "Geom 2" (Figures 8, 9, and 10).

The 1-D code can be assumed as a reliable tool for the prediction of the HTC related to the multiple jet section of an ACC cooling tube.

\section{Performance Analysis of Different ACC Cooling Tube Geometries}

The developed code has been used to evaluate the performance, in terms of the jet's mean HTC profile, of different ACC tube geometries.

7.1. Impact of the $A_{r}$ on the Jet Mean HTC Profile. It emerges from Figure 9 that the mass flow rate split across an ACC cooling tube can be largely variable. Andreini and DaSoghe [9] point out that the mass flow rate split across a given impingement system (i.e., the local MVR) is only related to the pipe area ratio $A_{r}$ defined as the ratio of the manifold inlet area and the holes cumulative exit area,

$$
A_{r}=\frac{A_{c}}{n_{\text {holes }} \cdot A_{j}} .
$$

Expression 1 demonstrates that, for each operating condition (i.e., for each $\beta$ ratio), the discharge coefficient is roughly constant when the MVR is higher than 5. As the MVR is a function of the $A_{r}$ parameter, it is possible to design the impingement system in order to assure that the mass flow rate through each hole is roughly the same. Indeed, assuming 


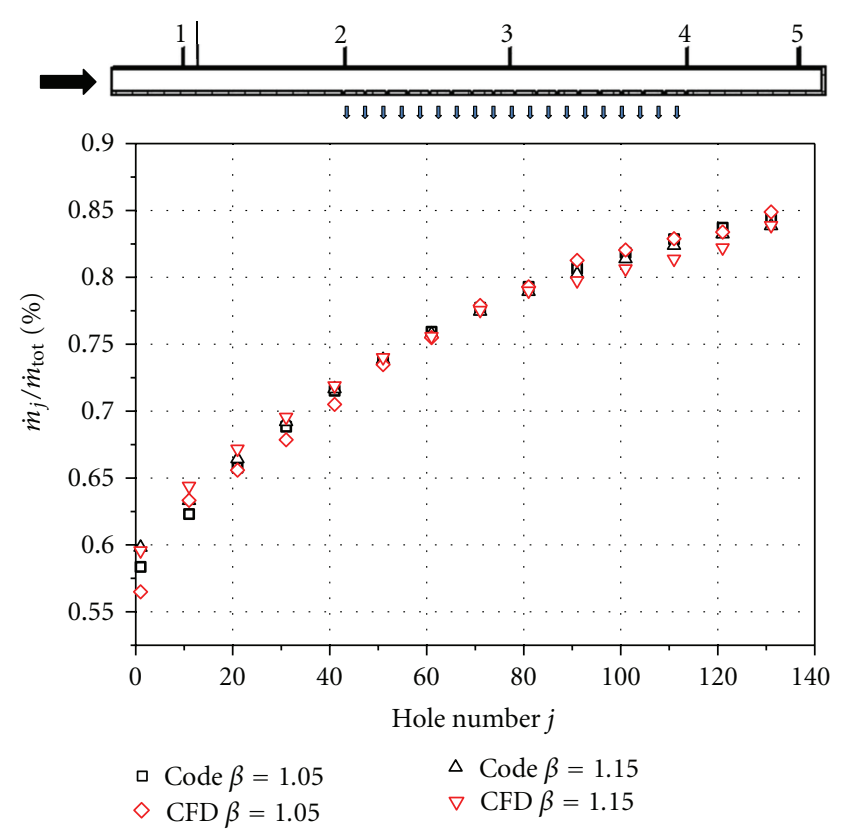

Figure 9: Mass flow split distribution for Geom 2 (percent).

that the manifold inlet mass flow rate is equally distributed among the impingement holes, it follows that

$$
\begin{gathered}
A_{c} \cdot(\rho \cdot v)_{c}=n_{\text {holes }} \cdot A_{j} \cdot(\rho \cdot v)_{j}, \\
\operatorname{MVR}=\frac{A_{c}}{n_{\text {holes }} \cdot A_{j}} .
\end{gathered}
$$

The relations above are satisfied when the $C_{d}$ is constant across the impingement system that is, when the MVR is higher than 5 approx. So it can be assumed that the condition:

$$
\frac{A_{c}}{n_{\text {holes }} \cdot A_{j}}=A_{r}>5,
$$

defines a design rule for manifolds with equally distributed impinging mass flow rate jets.

Geometries for which the $A_{r}$ is greater than 5 lead to a roughly constant impingement jet mass flow rate distribution, while, in case of geometries characterized by a low $A_{r}$, relevant differences in jet mass flow rates across the feeding pipe are expected. This last statement justifies the trends shown in Figures 5 and 9. Geom 1 is characterized by $A_{r}=8.4$ so the mass flow split across the manifold is uniform. In case of Geom 2, for which $A_{r}=1.08$, the impinging jet mass flow rate varies significantly from the first to the last hole of the array. It is also expected that in case of a low $A_{r}$, the impinging jet HTC could be nonuniform. Figure 11 shows the jet's mean HTC distribution obtained for Geom 2 when $\beta=1.05$. The parameter $\mathrm{HTC}_{\text {ref }}$ represents the mean heat transfer coefficient evaluated for the last hole of the array of Geom 2 when $\beta=1.05$. The figure reveals that the mean HTC varies from the inlet to the outlet of the manifold by about $25 \%$. This variation might be higher when considering high by-pass ratio turbofans.

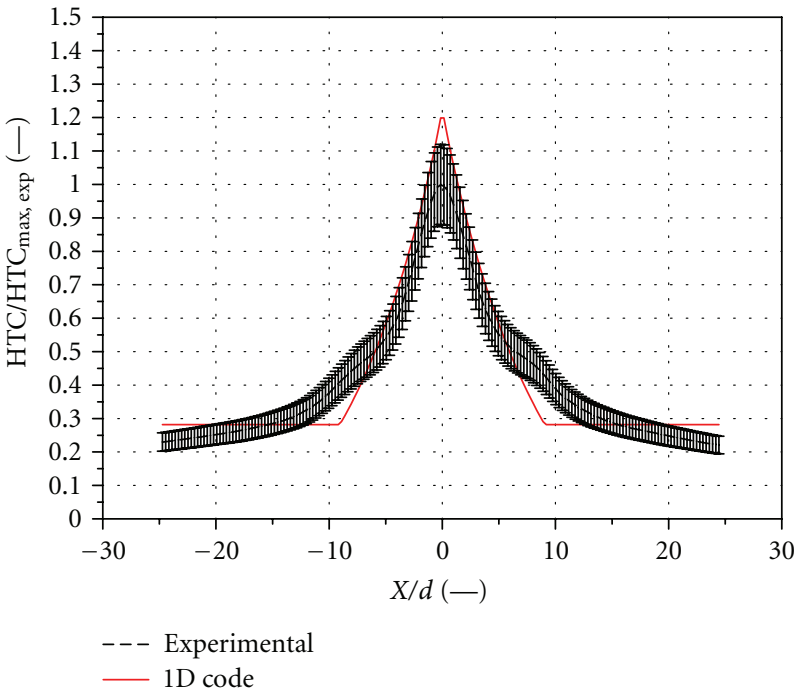

FIgURE 10: Heat transfer coefficient distribution, central hole of the array, Geom $1 \beta=1.05$ (experimental data from Facchini et al. [14]).

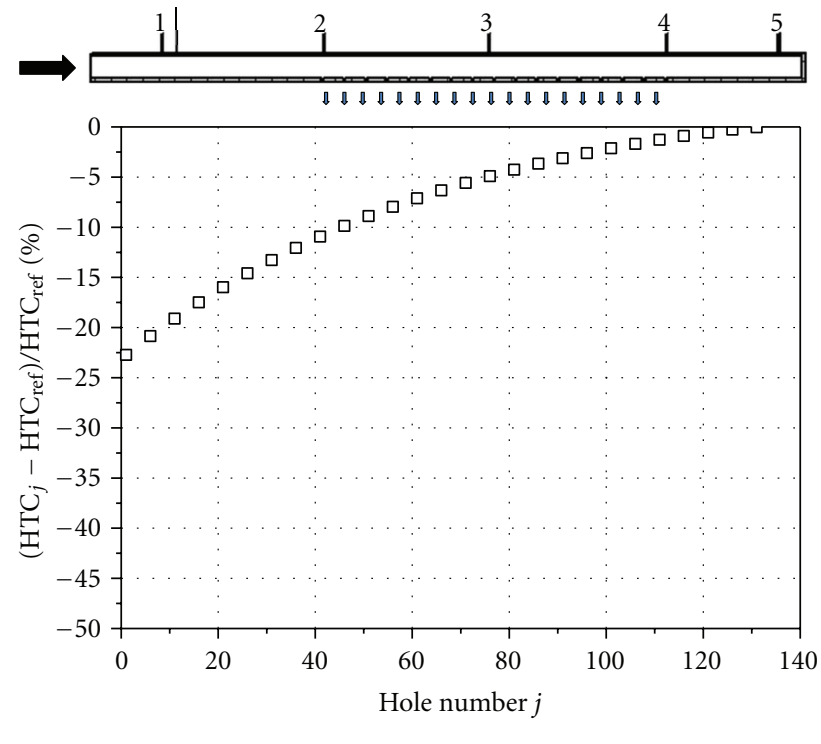

ㅁ $\beta=1.05$

Figure 11: Mean heat transfer coefficient distribution for Geom 2 $\left(A_{r}=1.08\right) \beta=1.05$.

7.2. Impact of the Manifold Cross Section on the Jet Mean HTC Profile. Figure 12 shows the jet mean HTC distribution along the manifold for different values of the $A_{r}$ parameter. The profiles are obtained assuming the same overall $\beta$ ratio $(\beta=$ $1.05)$, the same nozzle length-to-diameter ratio $(t / d=2)$, the same impinging holes diameter $d=1 \mathrm{~mm}$, and the same nozzles number (i.e., the only parameter that changes is the manifold diameter " $D$ ").

As discussed above, the mean HTC profiles are flatter as the $A_{r}$ increases. More in detail, the HTC profile's variations lie below $5 \%$ when the $A_{r}$ is higher than 3 . The better 


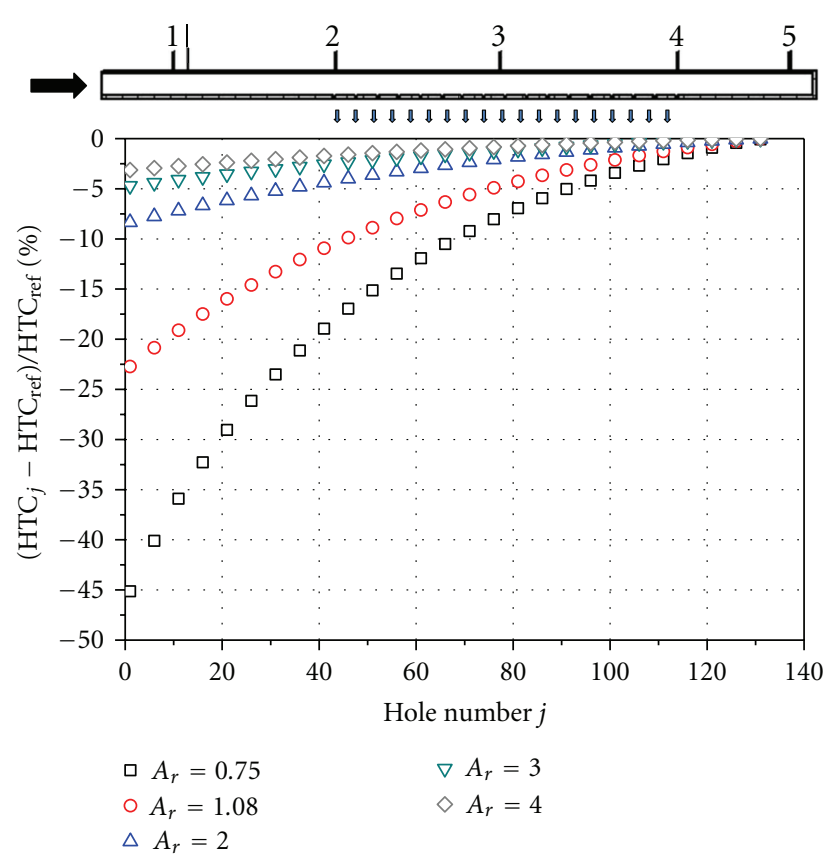

FIGURE 12: Mean heat transfer coefficient distribution varying the $A_{r}$ parameter, $\beta=1.05$.

performance obtained with the high $A_{r}$ is due to a more uniform mass flow rate split along the manifold. As the mass flow through last hole of the array remains roughly the same, the total mass flow at the manifold inlet increases when the $A_{r}$ is higher. Figure 13 shows the mass flow rate at the inlet of the feeding pipe for different $A_{r}$ values. The parameter $m_{\text {ref }}$ represents the total mass flow at the manifold inlet evaluated for Geom 2 when $\beta=1.05$.

When the $A_{r}$ is equal to 3 (i.e., when the changes in the HTC values along the manifold are below 5\%) the mass flow rate at the manifold inlet increases, with respect to the nominal case with $A_{r}=1.08$, by $9 \%$.

The increase of the $A_{r}$ by means of the manifold diameter " $D$ " augmentation, has a beneficial effect on the jet's HTC profile uniformity. However, it has to be remarked that as the feeding pipe diameter increases, both the cooling system size and weight increase. Furthermore, the total coolant mass flow rate is higher as well. The last evidence may have a marginal effect in case of cooling air extracted from the fan flow but represents a penalty if the coolant is bled from the compressor.

7.3. Impact of the $\beta$ Ratio on the Jet Mean HTC Profile. In present section, the effects of the overall $\beta$ ratio are analysed. The impact of the $\beta$ ratio on the jet's mean HTC profile is reported in Figure 14. To be consistent with the sensibility analysis previously conduced, the nozzles' diameter " $d$ " have been changed in order to assure, for each case, the same mean HTC value at the last hole of the array. The manifold diameter " $D$ " is fixed $(D=12 \mathrm{~mm})$ and the $t / d$ is equal to 2 . Thus, as the $\beta$ changes, the system $A_{r}$ consequently varies.

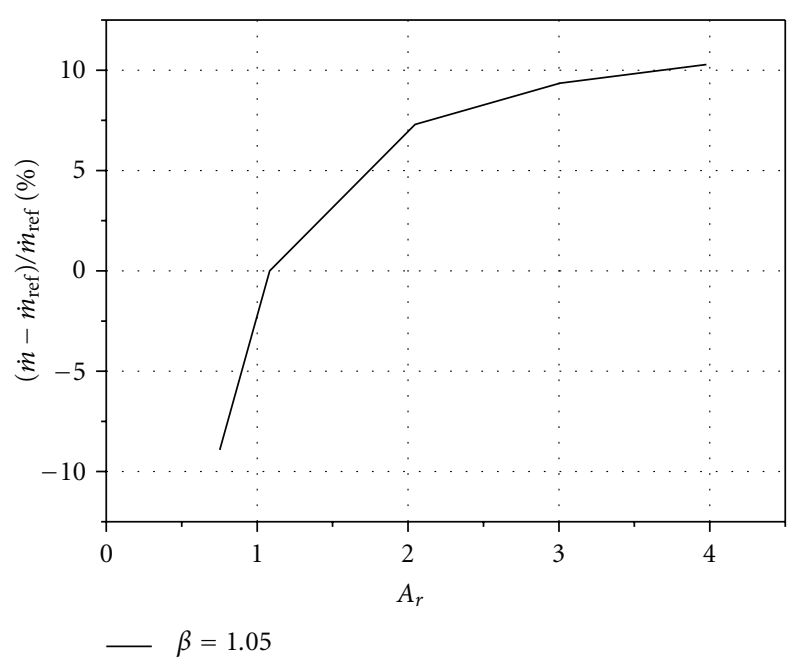

FIGURE 13: Total coolant mass flow rate varying the $A_{r}$ parameter, $\beta=1.05$.

Figure 14 shows that the increase of the system's overall pressure ratio has a beneficial effect on the HTC profile. This last statement can be motivated by observing that the $\beta$ ratio affects both the isentropic mass flow rate and the impingement holes' discharge coefficient (i.e., as the $\beta$ ratio increases both the $C_{d}$ and the $m_{\text {ideal }}$ result augmented). In order to obtain the same mean HTC value at the last hole of the array, the nozzles' diameter is then reduced. Thus the $A_{r}$ increases significantly as the design pressure ratio is increased explaining the trend shown in Figure 14.

The reduction of the impingement holes' cross section leads, as the pressure ratio is increased, to an appreciable reduction of the coolant cumulative mass flow rate (Figure 15).

When $\beta=1.2$, the HTC profile's variations lie below $5 \%$ and the total coolant mass flow rate is reduced by $13 \%$ with respect to the case when $\beta=1.05$.

7.4. Impact of the Nozzles' Diameter Distribution on the Jet Mean HTC Profile. In the previous sections, for each considered geometry, the nozzles' diameter is uniform moving from the first to the last hole of the array. However in order to mitigate the changes in the nozzles' mass flow rate, the impinging manifold could be designed considering a non-uniform holes' diameter distribution. The 1-D code is then used to design manifold geometries characterized by nozzles with different diameter. Each hole's diameter is determined imposing the same mean HTC value (i.e., the designed impinging manifold guarantee a uniform HTC level moving from the first to the last nozzle of the array). When considering a manifold diameter $D=12 \mathrm{~mm}, \beta=$ 1.05 , and $t / d=2$, the nozzles' diameter distribution across the manifold calculated by the $1-\mathrm{D}$ program is shown in Figure 16.

The first nozzle's diameter is increased by $80 \%$ with respect to the last one. For this manifold geometry, the calculated cumulative coolant mass flow rate is roughly 


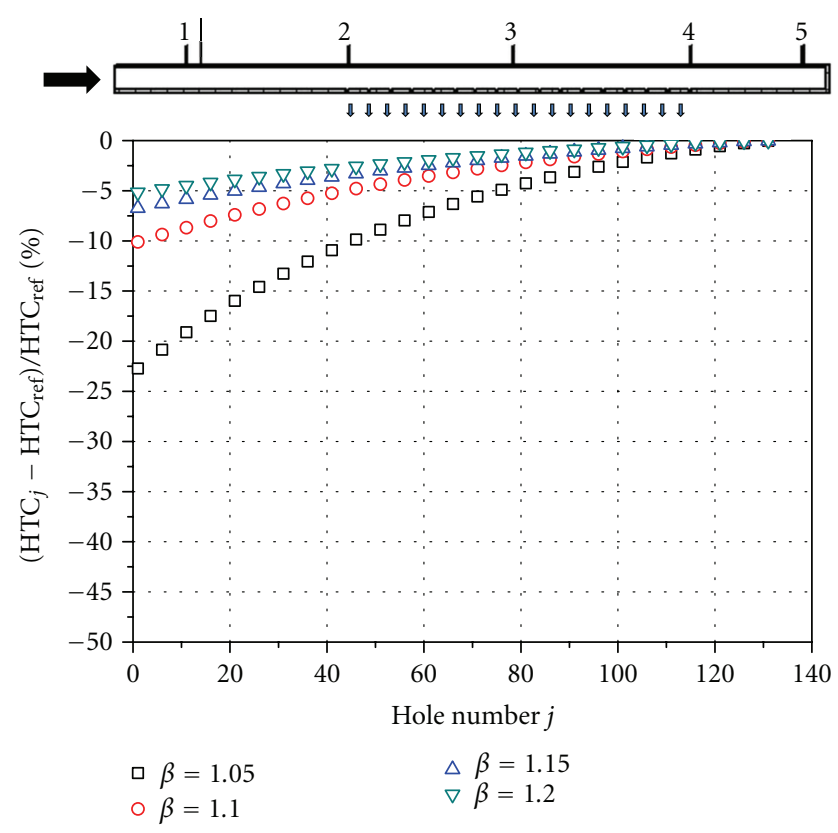

FIGURE 14: Mean heat transfer coefficient distribution varying the $\beta$ parameter.

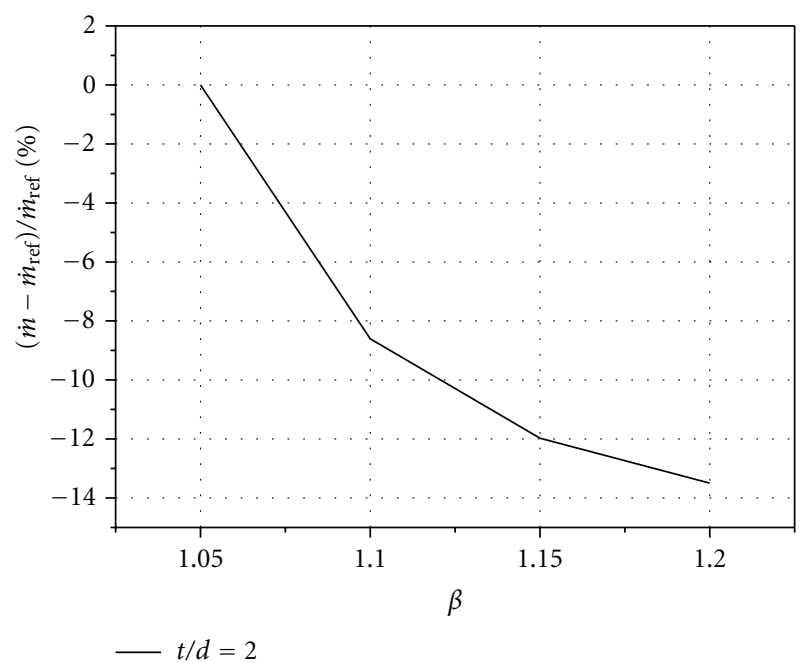

FIGURE 15: Total coolant mass flow rate varying the $\beta$ parameter.

$26 \%$ higher than those obtained in case of uniform holes' diameter $d=1 \mathrm{~mm}$.

Figure 17 reports the nozzles' diameter distribution calculated in case of different manifold diameter $D$. As expected, the changes in the impinging holes diameter is reduced as the feeding pipe cross section is augmented. The last evidence also results in a reduction of the total coolant mass flow rate, with respect to the case for which $D / D_{\text {ref }}=1$ (i.e., $D=12 \mathrm{~mm}$ ), Figure 18 .

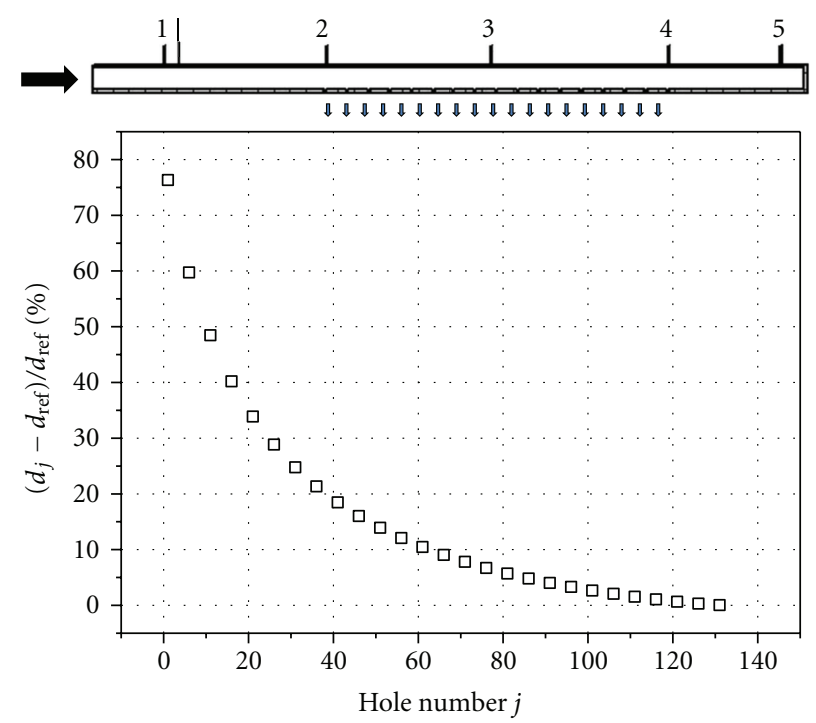

Figure 16: Nozzles' diameter distribution across the manifold $\beta=$ $1.05, D=12 \mathrm{~mm}$, and $t / d=2\left(d_{\mathrm{ref}}=1 \mathrm{~mm}\right)$.
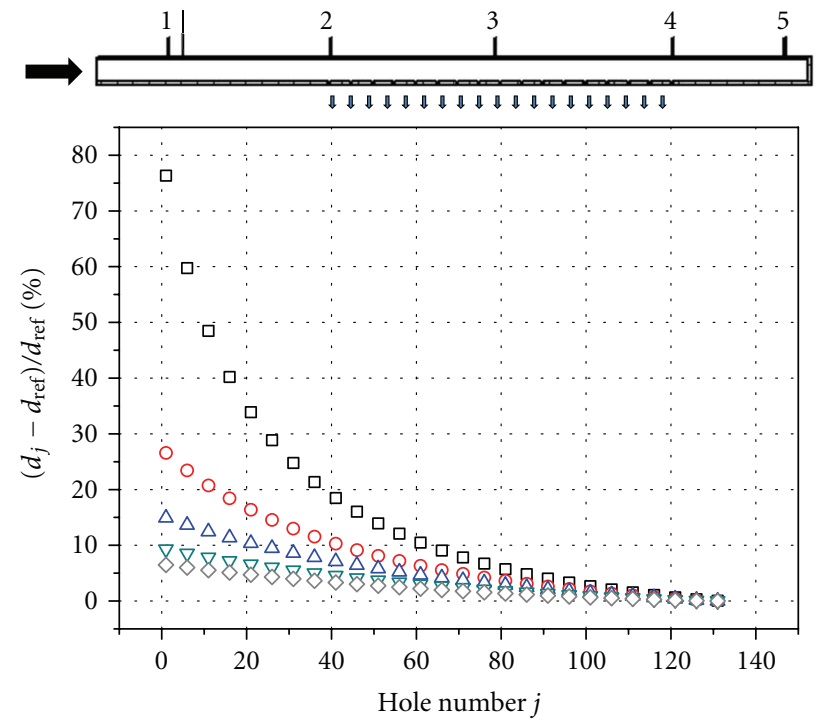
$D / D_{\text {ref }}=1$
$\nabla D / D_{\text {ref }}=1.5$
○ $D / D_{\text {ref }}=1.17$
$\diamond D / D_{\text {ref }}=1.67$
$\triangle D / D_{\text {ref }}=1.33$

Figure 17: Nozzles' diameter distribution across the manifold $\beta=$ $1.05, t / d=2$ varying the manifold diameter $\left(d_{\text {ref }}=1 \mathrm{~mm}\right.$ and $D_{\text {ref }}=12 \mathrm{~mm}$ ).

\section{Conclusions}

This paper deals with a simplified 1-D code aimed at the design of the multiple jet section of an ACC cooling tube. The $1-\mathrm{D}$ program is able to evaluate the mass flow rate through each nozzle of the array and then to calculate the related heat transfer coefficient (HTC) achieved on the target surface.

The code has been validated by means of both numerical and experimental data. Once validated, the program has been 


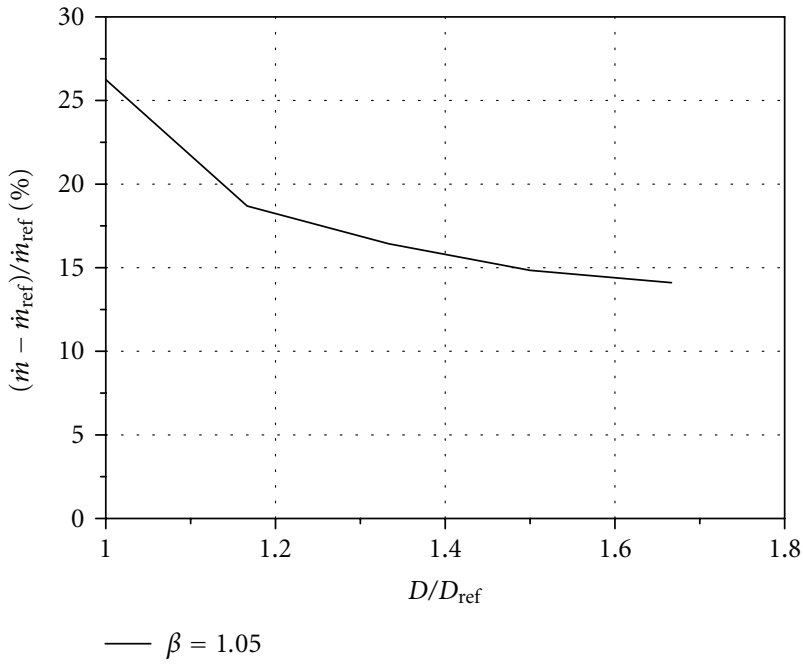

Figure 18: Total coolant mass flow rate varying the $D / D_{\text {ref }}$ parameter.

used to point out some design practices for such cooling systems.

It emerges from the study that the mass flow rate split across an ACC cooling tube can be largely variable. In that way, the key parameter is the pipe area ratio $A_{r}$ defined as the ratio of the manifold inlet area and the holes cumulative exit area. More in detail, geometries for which the $A_{r}$ is greater than 5 lead to a roughly constant impingement jet mass flow rate distribution while, in case of geometries characterized by a low $A_{r}$, relevant differences in jet mass flow rates across the feeding pipe are expected. Variations in the mass flow rate split across an ACC cooling tube result in HTC profile nonuniformities that should be avoided or reduced for a correct system design.

The augmentation of the pipe $A_{r}$ (by means of the increase of the manifold diameter) has a direct positive effect on the HTC profile uniformity. However, these solutions have few drawbacks: the increase of both the weight and size of the cooling system.

The role of the operating conditions on the manifold design has been considered. The calculations reveal that once designed considering high overall pressure ratio, the impinging system performs rather better. This last evidence has been motivated when observing that the increase of the pressure ratio leads to an augmentation of both the nozzles' discharge coefficient and the related isentropic mass flow rate. Thus, when imposing always the same mean HTC value at the last hole of the array, the increase of the $\beta$ ratio involves a higher pipe $A_{r}$. The coolant total mass flow rate is also reduced as the overall pressure ratio is augmented.

Finally, manifold geometry with non-uniform nozzles' diameter have been considered. In order to assure a uniform heat load, the impinging holes' diameter changes considerably across the manifold and the cumulative coolant mass flow rate is increased as well. In case of coolant air extracted from the fan flow, this kind of arrangement could be considered to limit the ACC system size and weight.

\section{Nomenclature}

$A_{c}$ : Tube internal area $\left(\mathrm{m}^{2}\right)$

$A_{j}:$ Impingement hole area $\left(\mathrm{m}^{2}\right)$

$A_{r}:$ Area ratio $A_{c} /\left(n_{\text {holes }} \cdot A_{j}\right)(-)$

$d$ : Cooling hole diameter $(\mathrm{mm})$

$d_{\text {ref: }}$ Cooling hole reference diameter $(1 \mathrm{~mm})(\mathrm{mm})$

$D: \quad$ Tube internal diameter $(\mathrm{mm})$

$D_{\text {ref: }}$ Tube internal reference diameter $(12 \mathrm{~mm})(\mathrm{mm})$

$h$ : Holes exit to target surface distance $(\mathrm{m})$

$k$ : Surface thermal conductivity $(\mathrm{W} / \mathrm{mK})$

L: $\quad$ Tube length $(\mathrm{mm})$

$\dot{m}$ : $\quad$ Mass flow rate $(\mathrm{kg} / \mathrm{s})$

Ma: Mach number (-)

$\mathrm{Nu}$ : Nusselt number HTCd/k(-)

$P$ : $\quad$ Pressure $(\mathrm{Pa})$

$\dot{Q}: \quad$ Heat flux $\left(\mathrm{W} / \mathrm{m}^{2}\right)$

$R: \quad$ Gas constant $(\mathrm{J} / \mathrm{kgK})$

Re: Reynolds number $(-)$

S: $\quad$ Pitch (mm)

$t$ : Nozzle length $(\mathrm{mm})$

T: $\quad$ Temperature $(\mathrm{K})$

$x$ : Distance between the considered point and the stagnation point $(\mathrm{m})$.

Acronyms

ACC: Active clearance control

$C_{d}$ : Discharge coefficient $(-)$

HTC: Heat transfer coefficient $\dot{Q} /\left(T_{a w}-T_{w}\right)\left(\mathrm{W} / \mathrm{m}^{2} \mathrm{~K}\right)$

MV: Mass velocity $\rho v\left(\mathrm{~kg} / \mathrm{sm}^{2}\right)$

MVR: Mass velocity ratio $(\rho v)_{j} /(\rho v)_{c}(-)$.

Greek Letters

$\beta$ : Global pressure ratio (P@manifold

inlet/P discharge) $(-)$

$\beta_{L}$ : Local pressure ratio (-)

$\mu$ : Air viscosity $(\mathrm{kg} / \mathrm{ms})$

$\rho:$ Air density $\left(\mathrm{kg} / \mathrm{m}^{3}\right)$.

Subscripts

av: Averaged value

aw: Adiabatic wall

c: Main channel

$d$ : Discharge conditions

exp: Experimental value

max: Maximum value

$n$ : Last hole of the array

is: Isentropic

$j$ : Jet impingement hole

ref: Reference value

$s: \quad$ Static

$t$ : Total 
$w$ : Wall

$x$ : Streamwise direction.

\section{Acknowledgment}

The authors wish to express their gratitude to F. Maiuolo and L. Tarchi for providing the experimental data.

\section{References}

[1] J. C. Han, J. S. Park, and C. K. Lei, "Heat transfer enhancement in channels with turbulence promoters," Journal of Engineering for Gas Turbines and Power, vol. 107, no. 3, pp. 628-635, 1985.

[2] J. F. Justak and C. Doux, "Self-acting clearance control for turbine blade outer air seals," in Proceedings of ASME Turbo Expo (GT2009-59683), pp. 1229-1237, June 2009.

[3] E. Halila, D. Lenahan, and T. Thomas, High Pressure Turbine Test Hardware, NASA CR-167955, 1982.

[4] B. Beck and W. Fasching, CF6 Jet Engine Performance Improvement-Low Pressure Turbine Active Clearance Control, NASA CR-165557, 1982.

[5] F. Ahmed, B. Weigand, and K. Meier, "Heat transfer and pressure drop characteristics for a turbine casing impingement cooling system," in Proceedings of the ASME International Heat Transfer Conference, IHTC14-22817, vol. 5, Washington, DC, USA, August 2010.

[6] F. Ahmed, R. Tucholke, B. Weigand, and K. Meier, "Numerical investigation of heat transfer and pressure drop characteristics for different hole geometries of a turbine casing impingement cooling system," in Proceedings of ASME Turbo Expo, GT201145251, pp. 1095-1108, Vancouver, BC, Canada, 2011.

[7] A. H. Lefebvre, Gas Turbine Combustion, Taylor \& Francis, 1998.

[8] N. Hay and D. Lampard, "The discharge coefficient of flared film cooling holes," in Proceedings of ASME Turbo Expo, 95GT-15, June 1995.

[9] A. Andreini and R. DaSoghe, "Numerical characterization of aerodynamic losses of jet arrays for gas turbine applications," Journal of Engineering for Gas Turbine and Power, vol. 134, no. 5, article 052504, 8 pages, 2012.

[10] M. Gritsch, A. Schulz, and S. Wittig, "Method for correlating discharge coefficients of film-cooling holes," AIAA Journal, vol. 36, no. 6, pp. 976-980, 1998.

[11] M. Gritsch, A. Schulz, and S. Witting, "Effect of internal coolant crossflow orientation on the discharge coefficient of shaped film cooling holes," in Proceedings of ASME Turbo Expo, 99-GT-40, 1999.

[12] D. A. Rowbury, M. L. G. Oldfield, and G. D. Lock, "A method for correlating the influence of external crossflowon the discharge coefficients of film cooling holes," Journal of Turbomachinery, vol. 123, no. 2, pp. 258-265, 2001.

[13] A. Schulz, M. Gritsch, and S. Wittig, "Effect of crossflows on the discharge coefficient of film cooling holes with varying angles of inclination," in Proceedings of ASME Turbo Expo, 2001-GT-0134, 2001.

[14] B. Facchini, F. Maiuolo, L. Tarchi, and D. Coutandin, "Heat transfer and effectiveness evaluation of multiple impingement jet arrays for an active clearance control system," ISAIF (ISAIF10-58), 2010.

[15] H. Martin, "Heat and mass transfer between impinging gas jets and solid surfaces," Advances in Heat Transfer, vol. 13, pp. $1-60,1977$.
[16] J. C. Han, S. Dutta, and S. V. Ekkad, Gas Turbine Heat Transfer and Cooling Technology, Taylor \& Francis, 1st edition, 2000.

[17] R. DaSoghe, B. Facchini, F. Maiuolo, M. Micio, and L. Tarchi, "Discharge coefficient characterization of jet array impingement holes for an active clearance control system," in Proceedings of the 9th ETC Conference, vol. 252, Istanbul, Turkey, March 2011.

[18] R. DaSoghe and A. Andreini, "Numerical characterization of pressure drop for turbine casing impingement cooling system," in Proceedings of ASME Turbo Expo, GT2012-68787, 2012.

[19] L. W. Florschuetz, C. R. Truman, and D. E. Metzger, "Streamwise flow and heat transfer distributions for jet array impingement with crossflow," Journal of Heat Transfer, vol. 103, no. 2, pp. 337-342, 1981. 

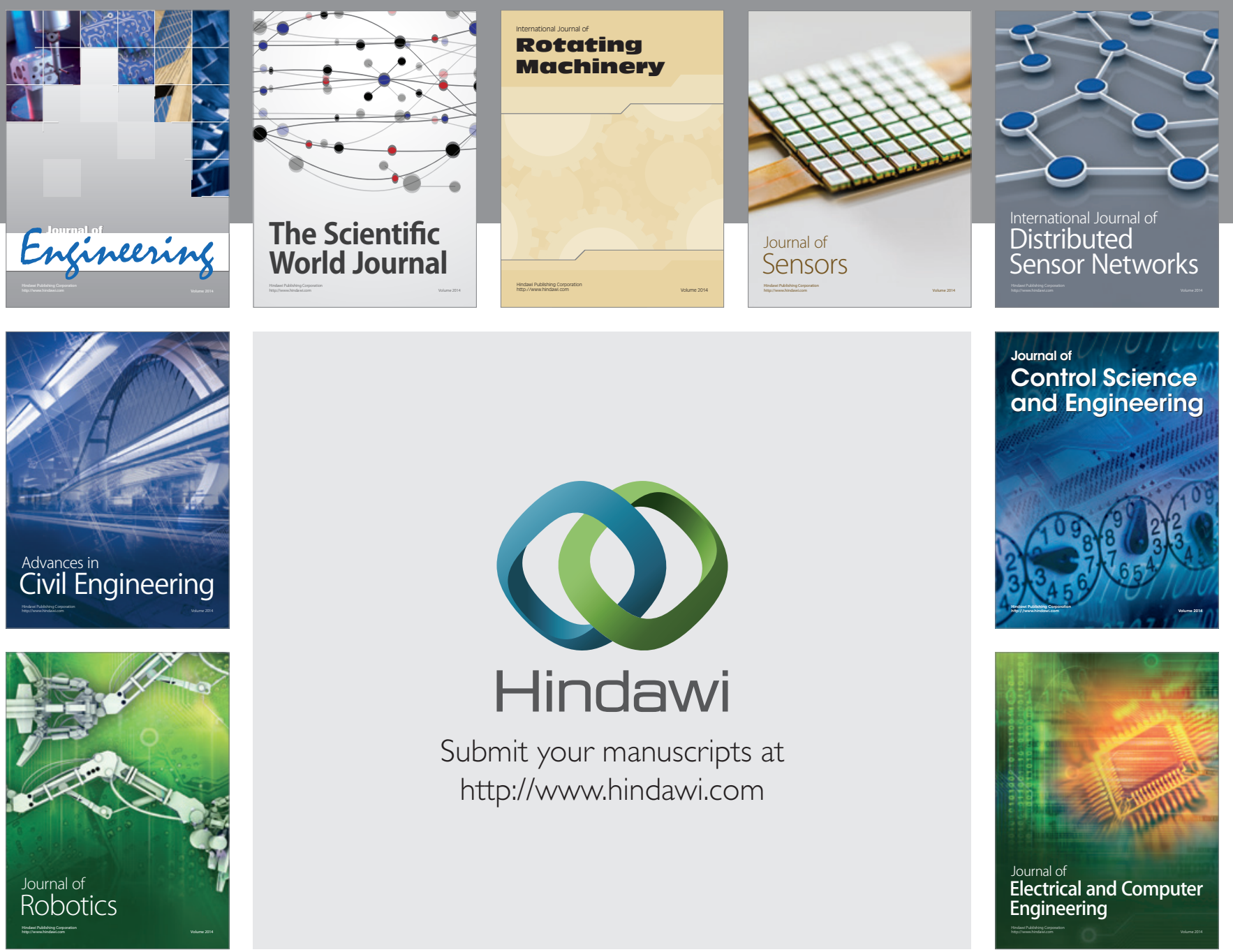

Submit your manuscripts at

http://www.hindawi.com
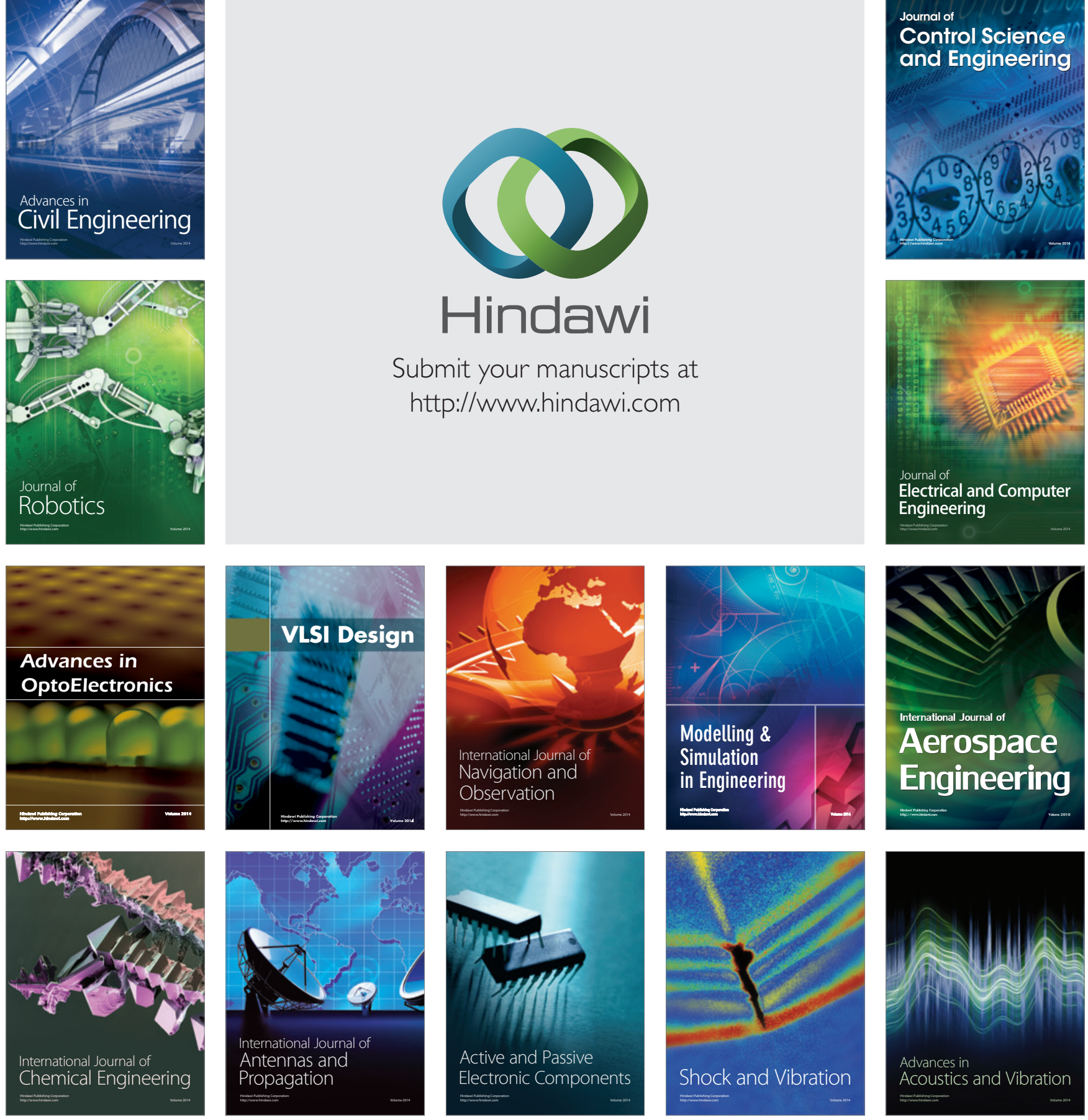